Reviews

have a general title please provide

\section{The phoneme}

McCully, Chris. 2009. The Sound Structure of English: An Introduction (Cambridge Introductions to the English Language) $264 \mathrm{pp}$. Hardback 978052185036 0, UK f45, US \$90; Paperback 978052161549 5, UK f15.99, US \$32.99;

Adobe eBook Reader 978051151258 2, \$26

Review by Philip Shaw, Stockholm University

This second volume in the Cambridge Introductions to the English Language series tackles the crowded market for introductory undergraduate textbooks in English phonetics and phonology. All the books on this market explain the basic terms and facts and try to introduce the complex idea of the phoneme, so new entrants have to

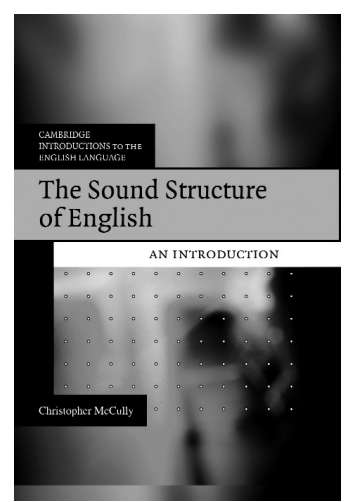
identify a niche that differentiates their product. McCully's unique selling points are an intellectual, phonology-first approach, inclusion of diachronic aspects, and an apparent commitment to plurivocality managed by a highly-present author. Much space is devoted to the structure of the syllable nucleus, but almost none to that of the larynx, for example. Saussure, Strang, Gimson, Crystal, Roach, McMahon and many others are cited and quoted freely but $I$, the writer, is also always present, telling anecdotes to you the reader, explaining, commenting on progress, encouraging, even warning. This busy involvement with the reader is also helpfully manifested in chapter previews, boxed comments, hints and open questions (with very helpful answers on the book's website), endof-chapter exercises, checklists of key terms (with a final glossary), and frequent recommendations for further reading.

There are eleven chapters. After an introduction on writing and speech, accent and dialect, and the systematicity of language, three chapters deal with consonants, The first establishes the consonant inventory of English by means of substitution frames and minimal pairs, and starts to clarify the relation of sounds to spelling. The second introduces articulatory categories, emphasizing what readers can confirm by experience in their own mouths, and the third introduces allophones and complementary distribution by means of aspiration. The next three chapters deal with syllables, chapter 5 introducing phonotactics and stress, and 6 and 7 developing syllable structure and the constraints on it, including issues like the sonority hierarchy syllabification and ambisyllabicity. Chapters 8 to 10 then deal with vowels, although concepts like long and short and diphthong have had to be introduced to deal with syllable structure. Chapter 8 introduces the short vowel inventory the vowel trapezium and cardinal vowels. Realizations are exemplified from native varieties, eight from the 'British Isles', three from the US, and two from the Southern hemisphere. Chapter 9 covers long vowels and diphthongs, introducing rhoticity. Chapter 10 discusses historical splits and mergers and chain shifts and looks at the development of and variation in STRUT, TRAP and happY. Finally Chapter 11 moves to a (still) more theoretical level and introduces optimality theory.

McCully is keen to get to the phonologically interesting issues, but explains the basic phonetics well, with frequent appropriate warnings of common mistakes and misunderstandings. The open questions in the text are often difficult to interpret, but always well explained on the website, so that readers do not feel left in doubt. The plurivocality creates a sense in the reader that knowledge is constructed in a rational way and leads naturally to the useful wider reading recommended. The persona presented as $I$ is friendly and unpretentious. The wide range of exemplification of phoneme realizations is useful and the late and skeptical introduction of RP will help readers to feel that it is actually true that all accents are equal. The inclusion of an introduction to optimality theory is welcome and the exposition clear.

This is not, however, a book I can imagine assigning for any student readership, either for an initial one-term module (as the blurb suggests) or for a phonology course for those with a knowledge of the basic phonetics of English. In contrast to many competing publications, there is no $\mathrm{CD}$ and there are no recordings on the website. Availability of such recordings would have made the detailed discussion of variants of TRAP and happY, for example, much easier to follow. The focus is on non-rhotic, British, native varieties, and non-native students who have been exposed to a lot of US speech (through the media for example) and are themselves users of a mixed variety may find this confusing or irrelevant, although McCully teaches in the Netherlands and must have used this material with exactly such students.

The plurivocality breaks down at exactly the moments when things become complicated, and we are left with bald unexplained claims that may 
be well-founded but are delivered ex-cathedra. Thus in ban ( $\mathrm{p}$ 97) and impish (website, answer to ex 7A), and in grind (p 95) the nasal is in the coda, but in hint and imp (p 96, 99) it is in the nucleus. This issue is discussed at some length (without any reference to sources or differing analyses) but the underlying principle is never stated, and since we are working at a level where the spelling and pronunciation of diphthong can be explained in a box ( $\mathrm{p} 98$ ), one could reasonably expect this. The discussion of contrastive and complementary distribution leads to the assertion that the glottal stop is not an allophone of /t/ (p 57), although it is a realization of it. This claim is also delivered without reference to contrasting views, and I have not found a definition of realization in the book that would explain it to a confused student. Despite the highly abstract view of the language system evoked on page 9 (see discussion of transcription conventions on $\mathrm{p}$ 13), changes like the lowering of the realization of TRAP in RP over the last fifty years are, without discussion, described as changes of phoneme "RP /æ/ > /a/" (e.g. p 165). On page 166 the FOOT vowel is described as 'allophonic' with respect to STRUT, with a reference to a discussion that does not prove this for the definitions that McCully used for phonemes and allophones in earlier chapters. Attentive readers with an interest in the theoretical aspects of phonology - McCully's apparent target group - will be frustrated by these obscurities, of which I can only give a few examples.

Although the book would have to be used with great caution as a set text, it is worth reading for every teacher of English phonetics and phonology who would like a fresh approach and would like to benefit from noting all the pitfalls for beginning students that the author has pointed out. 